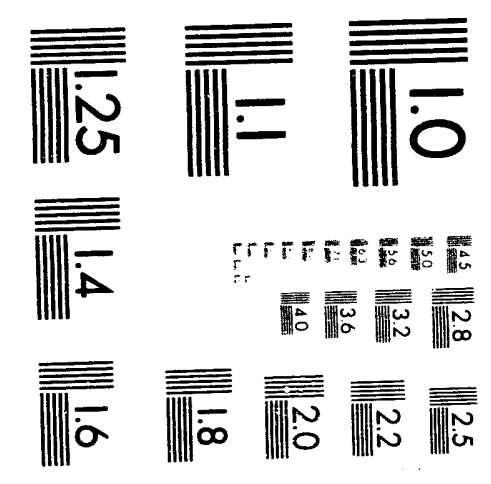



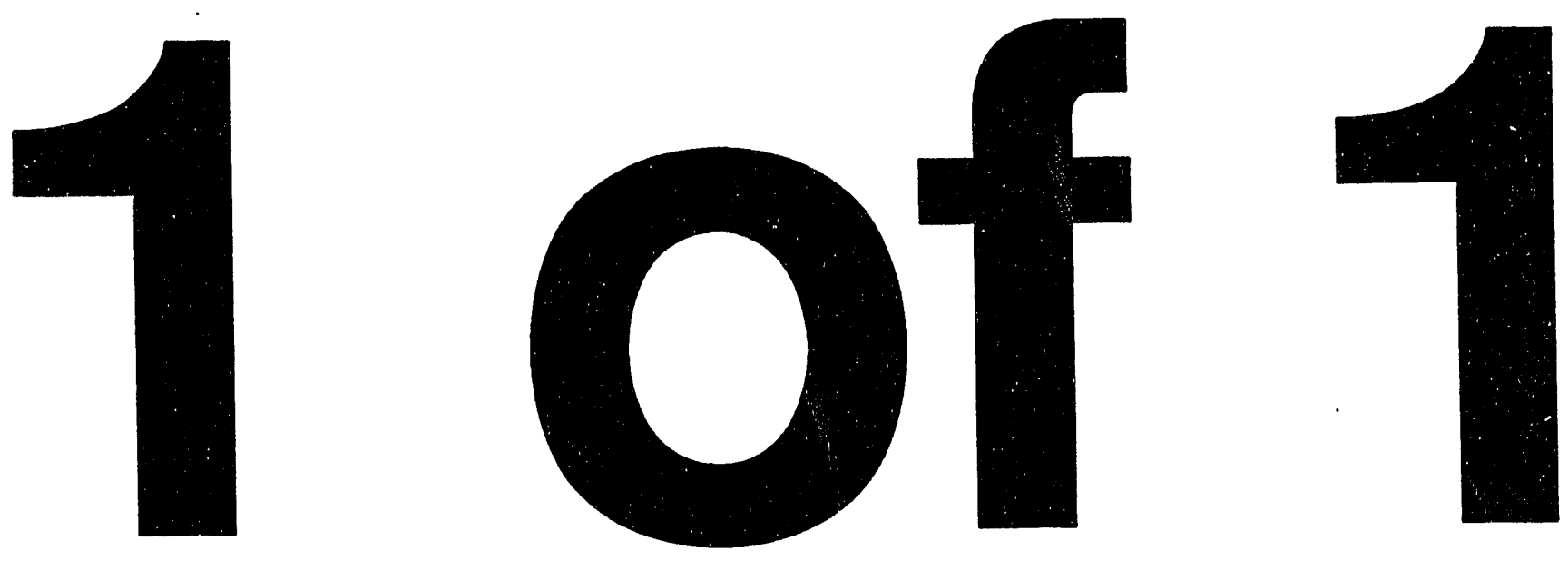


\section{Beam Line Error Analysis, Position Correction, and Graphic Processing}

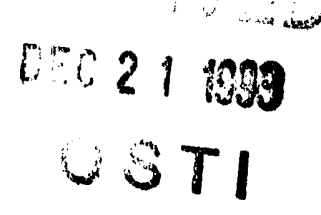

F. Wang and N. Mao

Superconducting Super Collider Laboratory*
2550 Beckleymeade Ave.
Dallas, TX 75237

November 1993

*Operated by the Universities Research Association, Inc., for the U.S. Department of Energy under Contract No. DE-AC35-89ER40486. 


\title{
Beam Line Error Analysis, Position Correction, and Graphic Processing
}

\author{
Fuhua Wang and Naifeng Mao
}

\begin{abstract}
A beam transport line error analysis and beam position correction code called "EAC" has been developed in association with a graphics and data post-processing package for 'TRANSPORT. Based on the linear optics design using TRANSPORT or other general optics codes, EAC independently analyzes effects of magnet misalignments, and systematic and statistical errors of magnetic fields, as well as the effects of the initial beam positions on the central trajectory and on the transverse beam emittance dilution. EAC also provides an efficient way to develop beam line trajectory correcting schemes.

The post-processing package generates various types of graphics including beam line geometrical layout, plots of the Twiss parameters, and beam envelopes. It also generates an EAC input file, thus connecting EAC with general optics codes. EAC and the post-processing package are small codes that are easy to access and use. They have become useful tools for the design of transport lines at the Superconducting Super Collider Laboratory.
\end{abstract}




\subsection{INTRODUCTION}

Nowadays we rely on popular general optics codes such as TRANSPORT to design beam line optics. However, it is always a desire of beam line designers to have handy tools to look at various aspects of the design, e.g., sensitivity to errors, basic trajectory correction schemes, etc. These "tool" codes should be easy to access and to modify to accommodate various real situations. Our work was performed in two steps. First, a TRANSPORT post-processing package was developed, enabling us to graphically examine the optical design of the line in various aspects, such as creating plots of Twiss functions, beam envelopes, dispersion vectors, and geometrical layout. These are extremely useful in the iteration process of lattice design. The next step was the development of the EAC code $^{1}$ for error effect analysis and graphically interactive beam position correction scheme design. An EAC input file is automatically generated during the normal TRANSPORT post-processing. Standard drawing software using "TOFDRAWER" is then applied. Users familiar with FORTRAN need expend little effort to i:se these graphic packages or to make their own favorite drawings by editing or imitating these codes. We confined ourselves mainly to linear optics and limited the scope of our work to keep the whole program package small.

\subsection{TRANSPORT POST-PROCESSING PACKAGE}

This package processes TRANSPORT output in either accelerator notation or traditional notation, which contain all necessary data for tach physical element along the beam line. It generates three standard data files for further processing. One includes details of element parameters, such as element names, lengths, apertures, fields, gradients, etc. The second contains various beam parameters, such as $\beta, \alpha, \eta, \psi$ functions. The third is a comprehensive file as the direct input of the following application codes. Figure 1 is a flow chart that depicts the main functions of this package.

A graphical code, BAEFSYN, along with a title file, BAETITLE.DAT, creates some TOPDRAWER files for plotting $\beta, \eta, \psi$ functions and beam envelopes along the beam line. The phase advance function $\psi\left(\psi_{x}\right.$ or $\psi_{\mathrm{y}}$ ) is plotted with $\psi-\mathrm{n} \pi / 2$ as ordinate. As an example, a plot for $\eta$ and $\beta$ functions of the Superconducting Super Collider (SSC) Low Energy Booster (LEB) to Medium Energy Booster (MEB) transfer line is shown in Figure 2. The GPXA and GPYA codes, along with two title files, GPXATITLE.DAT and GPYATITLE.DAT, create two TOPDRAWER data files, GPXA.TOP and GPYA.TOP, for beam line two-dimensional geometric layout plotting in both plane view and elevation view. An elevation view of the LEB to MEB transfer line and dump line layout is shown in Figure 3. Meanwhile, a three-dimensional coordinate data file, COD.DAT, is created by code CO3SYN. Coordinates at both ends for each element are given in this file. Code FSCCM creates a file FOR002.DAT, which will be the input data file for EAC.

This post-processing package is written in FORTRAN. The total length of the above major processing and application codes is about 1600 lines. 


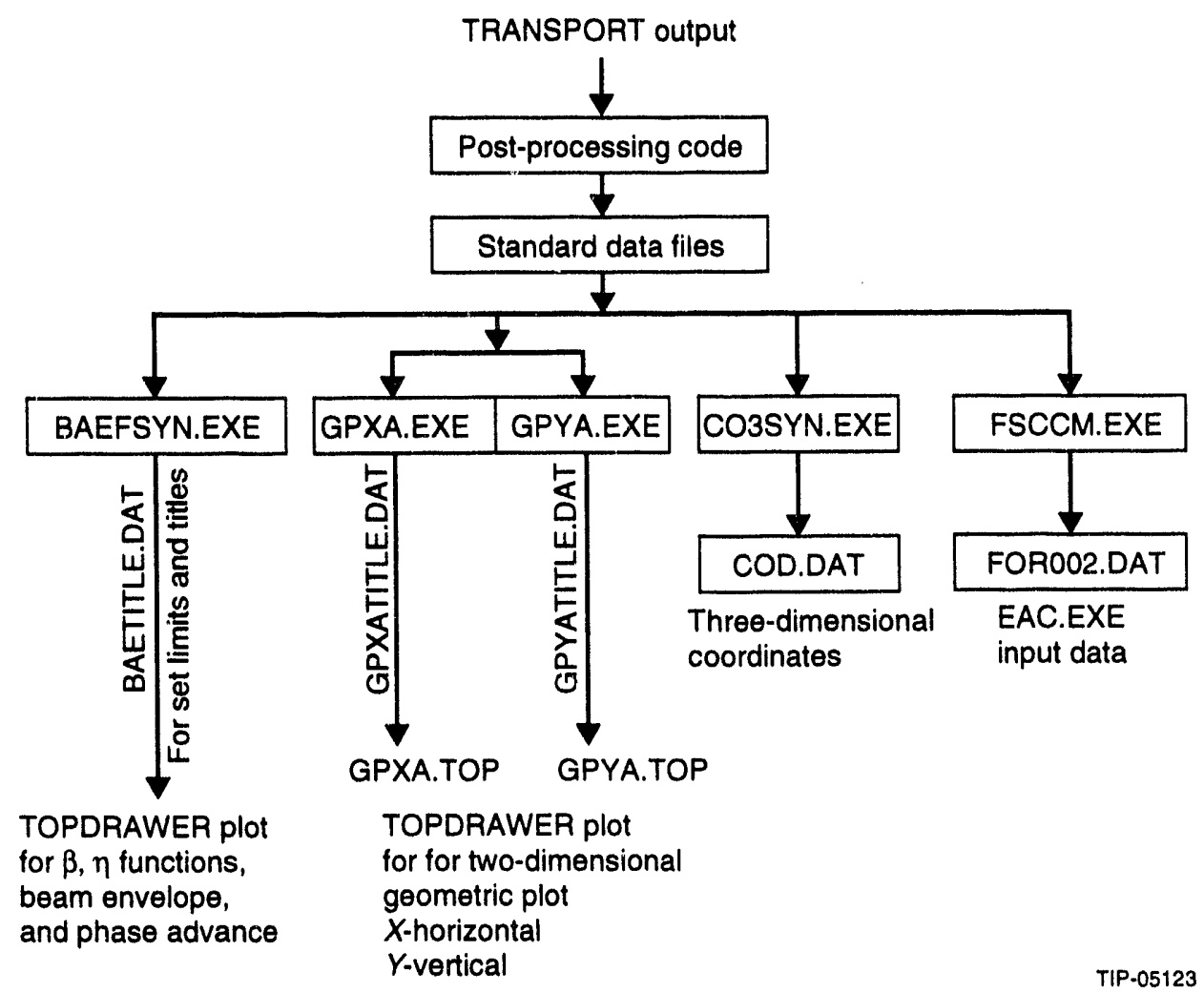

Figure 1. TRANSPORT post-processing package.

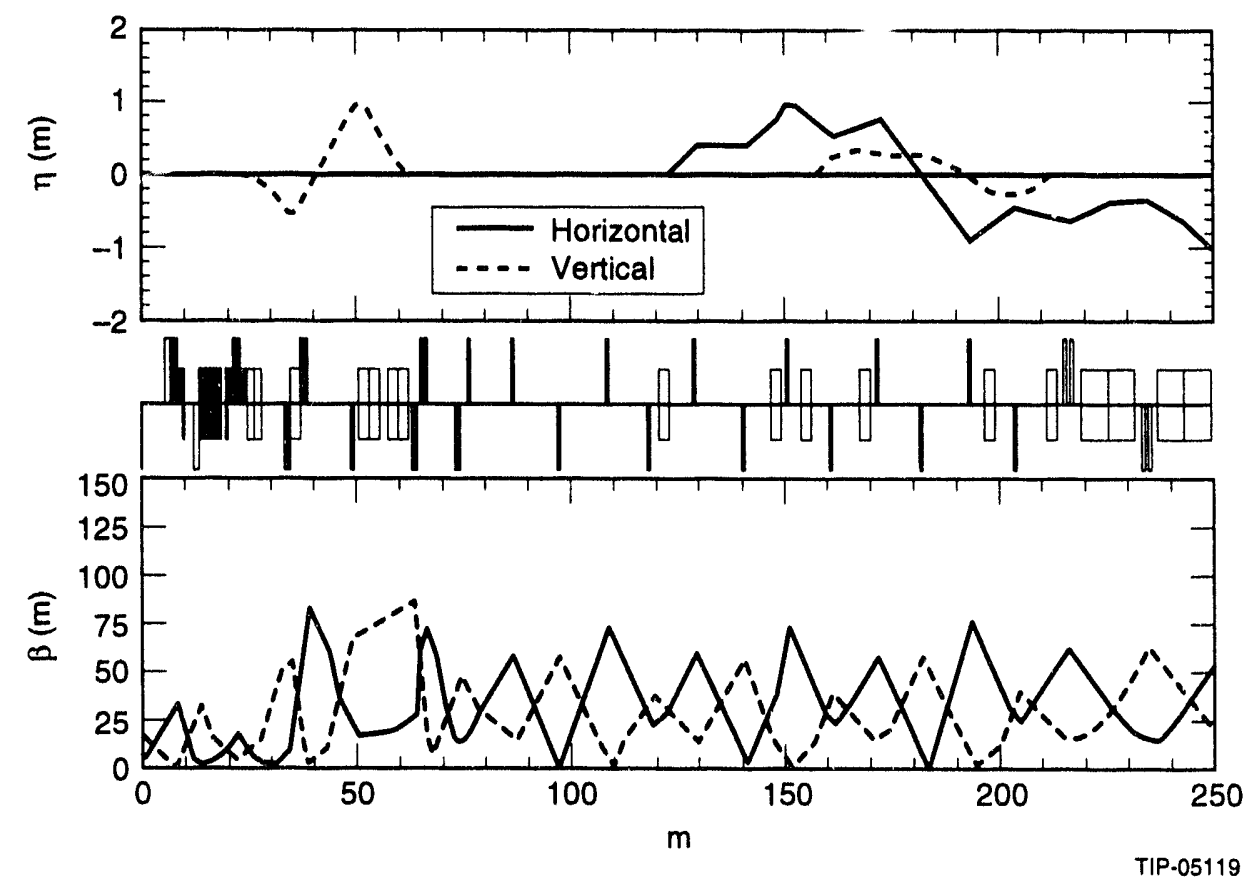

Figure 2. $\beta$ and $\eta$ functions. 


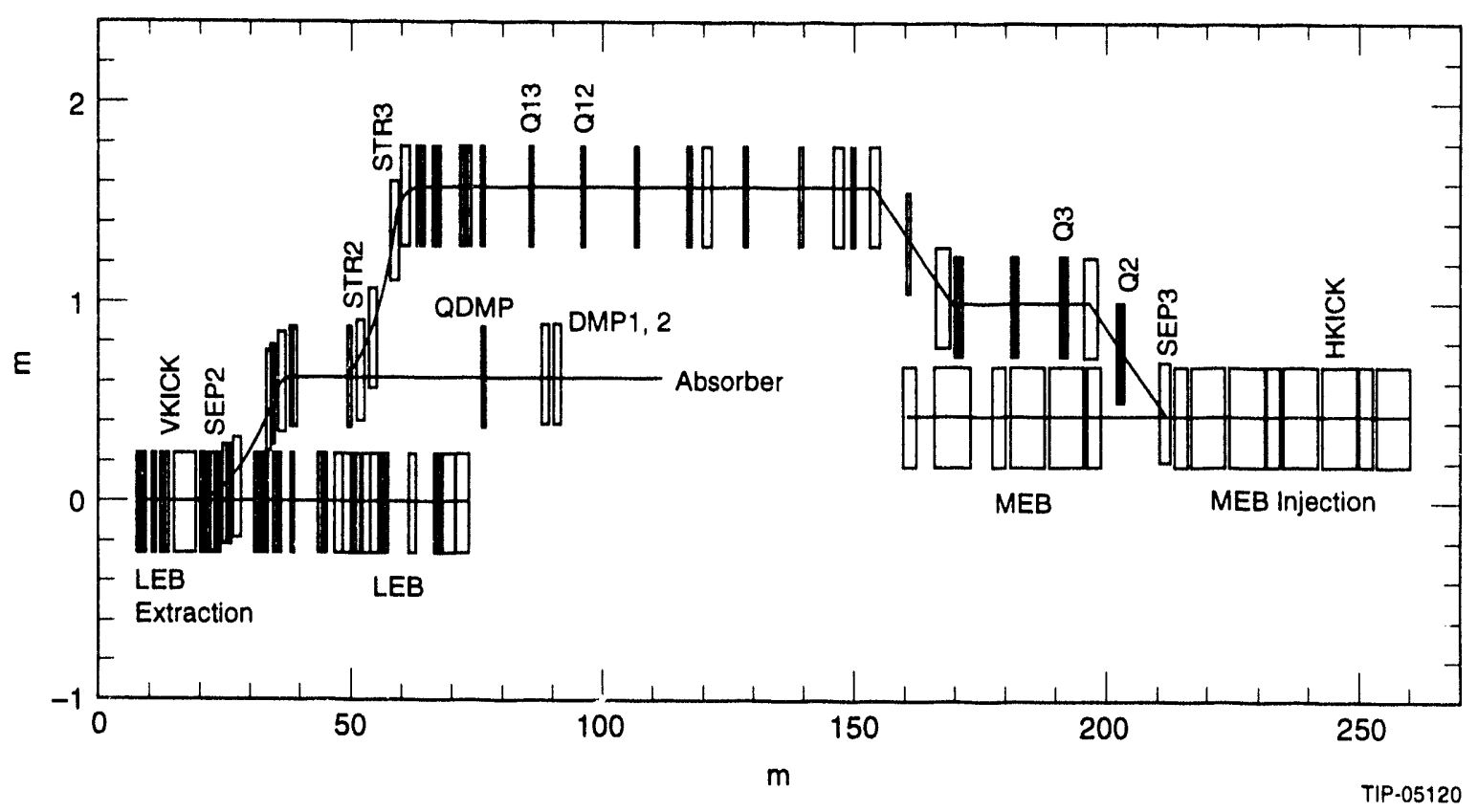

Figure 3. Layout of SSC LEB-MEB transfer and dump lines (elevation view).

\subsection{ERROR ANALYSIS AND POSITION CORRECTION CODE "EAC"}

The main functions of EAC are linear beam optics calculations, error effect analysis on the beam central trajectory, the beam phase space ellipse distortion, and the beam position correction scheme. A flow chart of $\mathrm{EAC}$ and its associated application codes are shown in Figure 4.

\subsection{Linear Beam Optics}

Linear beam optics is a useful tool for input data verification. Both graphical and digital outputs of optics are provided.

\subsection{Beam Trajectory Calculation}

EAC simulates the single-particle trajectory in the presence of various errors. For magnet misalignment effects, the mathematical base of TRANSPORT ${ }^{2}$ is adopted. Six-dimensional magnet displacements are taken into account. Other input errors are the beam initial position errors and bending magnet field errors. All errors can be input directly or generated by the code (random normal distribution). Magnet misalignment and other errors are uncertain in the design stage; multi-trial simulations on beam lines with random error distributions are applied to determine the tolerances on these errors. The program can assign all these errors from normal distributions with different standard deviation rms values. In this case, rms values for different types of errors are required for input.

Magnet misalignments, field errors, and initial beam position errors are responsible for beam centroid displacements from the reference trajectory along the beam line and for the closed orbit mismatching at injection.

Two criteria for tolerance setting may be appropriate: beam line aperture limits and closed orbit mismatching limit. Closed orbit mismatch will cause beam transverse emittance dilution to follow in the accelerator. Closed orbit mismatch caused by systematic errors can be reduced dramatically by a trajectory correction scheme. While orbit mismatch caused by time-related statistical errors can't be eliminated by a slow correction process, they should be restrained by regulation tolerance set to transfer line magnet power supplies. 


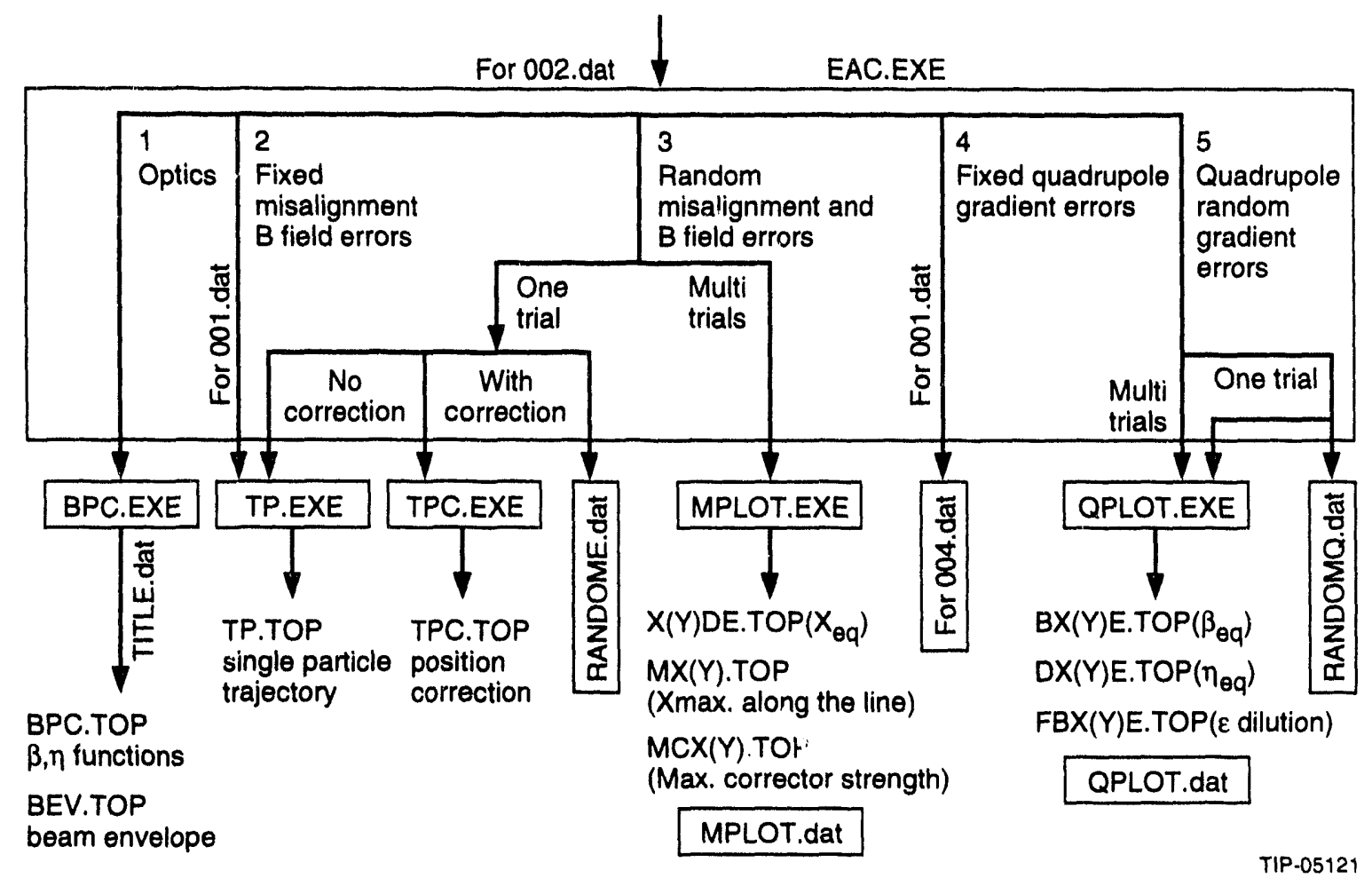

Figure 4. EAC program package.

Magnet field variation caused by current ripple and other time-related current variations may be coherent if they are powered in series. An option is provided to group magnets in the input data, so that magnets in one group will have the same field variation in such analysis.

The output of a single trial includes the beam positions (a data file and a plot, Figure 5) and error information (RANDOME.dat). The output results from a multi-trial run are: (1) distribution of maximum displacement along the line (Figure 6(a)), and (2) equivalent displacement $X_{\text {eq }}$ at the concerned point. $X_{\text {eq }}$ is a quantity used in determining possible phase area filamentation to follow in the accelerator. ${ }^{3}$

Toibulated results are given in file Mplot.dat. One can track back to any of the individual cases with graphics of central trajectory and detailed digital results by selecting the corresponding error seed number (see Figure 5).

\subsection{Beam Position Correction}

There are two correcting methods available in this code: ${ }^{2}$ one (corrector) to one (monitor) mode, and two (correctors) to two (monitors) mode (orthogonal control). The first way is easy for operating and may cost less. It is used for rough correction, which is sufficient in most of the line. The second method is used in cases where strict position error control is needed.

The error analyzing calculation can be performed with or without a correction scheme. When working with a correction scheme, information of beam position monitors and correction dipoles is needed for input. Monitors and correctors can be defined to work for one transverse direction or for both; correctors can be independent dipoles or auxiliary windings on magnets, depending on the need in real situations. There is more physics than programing for the arrangement of a correction scieme; however, graphically interactive iteration greatly helps the designer to understand the physics and take care of real requirements. 
The output of a multi-trial run with a correction scheme includes information on corrector strengths and other information similar to a non-correction run.

The designer can run a case of a single trial to start, and make a first fit of a correcting scheme with the help of a graphic display of the central trajectory. Then by examining the results of a multi-trial run, he can track and improve individual undesired cases. The design goals are to meet position error limit requirements and to simplify the correcting scheme to the extent possible, thereby reducing cost through the use of fewer correctors and monitors.

Figure 5 is an example of central trajectories before and after correction. Figure 6 is the statistical distribution of maximum beam displacement along the line and corrector strength needed for the above correcting scheme.

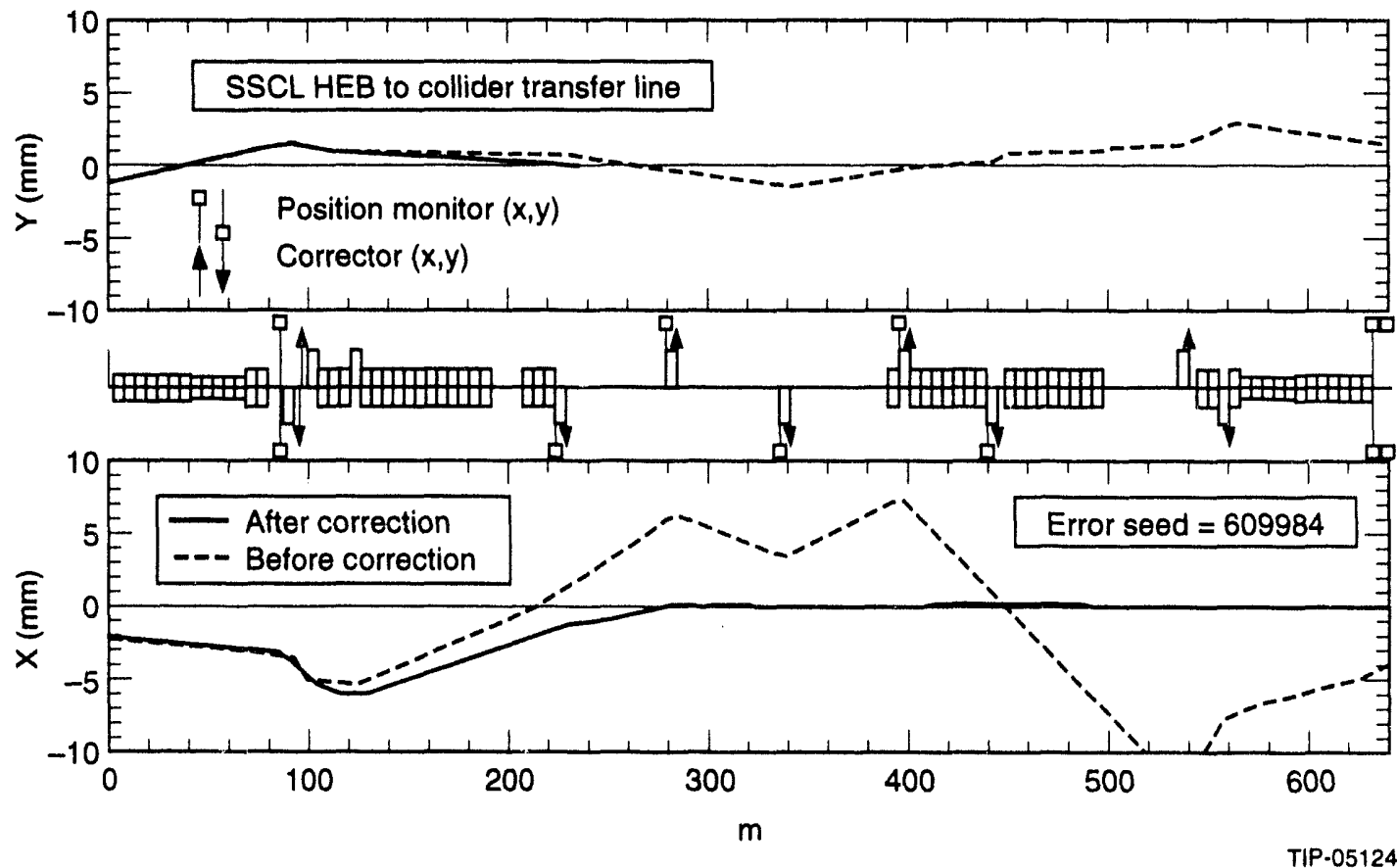

Figure 5. Beam position correction. 
(a)

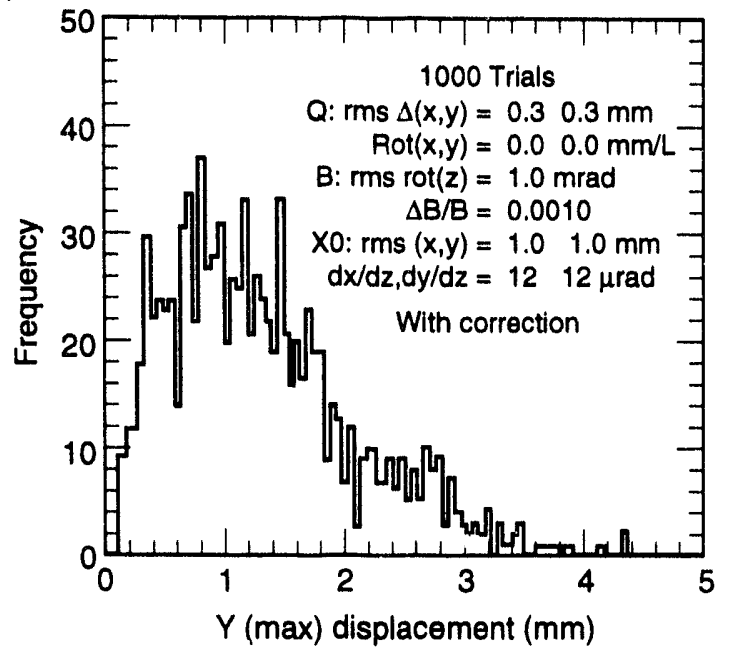

(b)

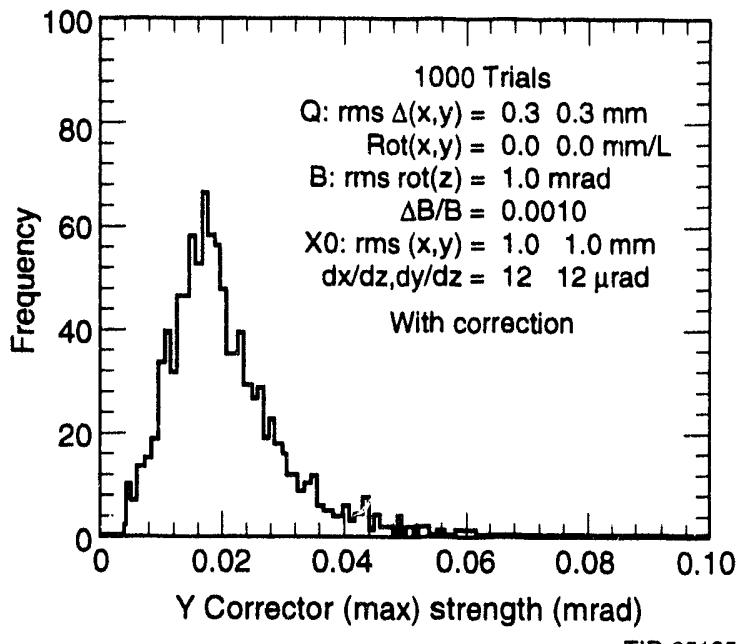

Figure 6. Maximum beam centroid displacement and corrector strength.

\subsection{Quadrupole Gradient Errors}

Quadrupole gradient errors will cause distortions of the beam emittance ellipse in phase space. The filamentation of the ellipse will lead to emittance dilution and should be reduced to a certain amount. The flow chart of this analysis is also given in Figure 4. One example is given in Figure 7 . The definition of equivalent $\beta$ function $\beta_{\mathrm{eq}}$ is again like $X_{\mathrm{eq}}$, a quantity directly related to emittance dilution. ${ }^{3}$

(a)

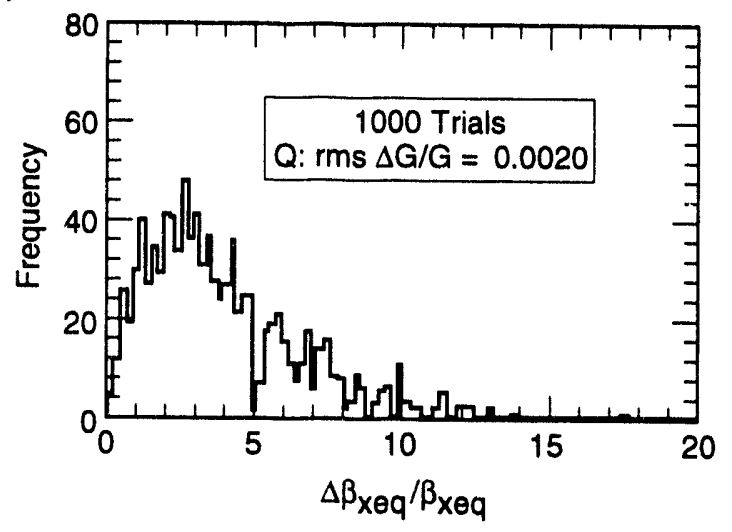

(b)

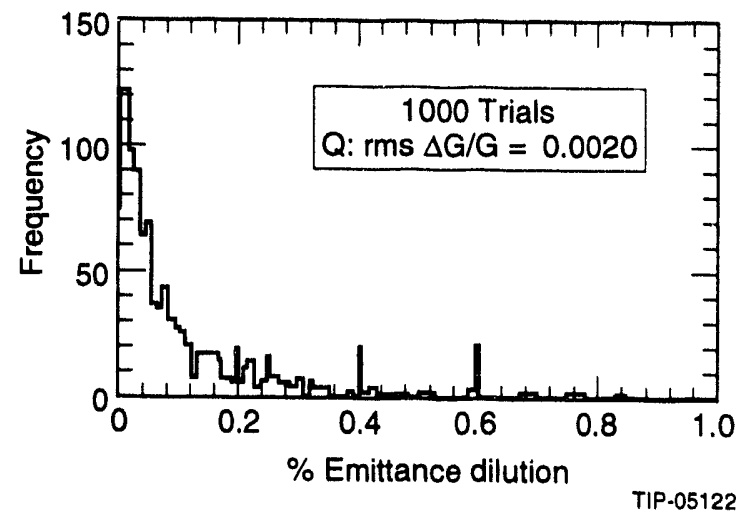

Figure 7. Sensitivity to gradient errors.

EAC code has about 2000 standard FORTRAN lines. 


\section{ACKNOWLEDGEMENTS}

We would like to thank Prof. K. Brown, Dr. J. McGill, and Dr. D. Johnson for their continuous support of this work. 


\section{REFERENCES}

1. Fuhua Wang, "EAC user's guide," SSCL internal memo, 1991.

2. Karl L. Brown et al., CERN 80-04, p. 229-243, 1980.

3. Michael J. Syphers, FNAL-TM-1456, p. 119, 1987. 

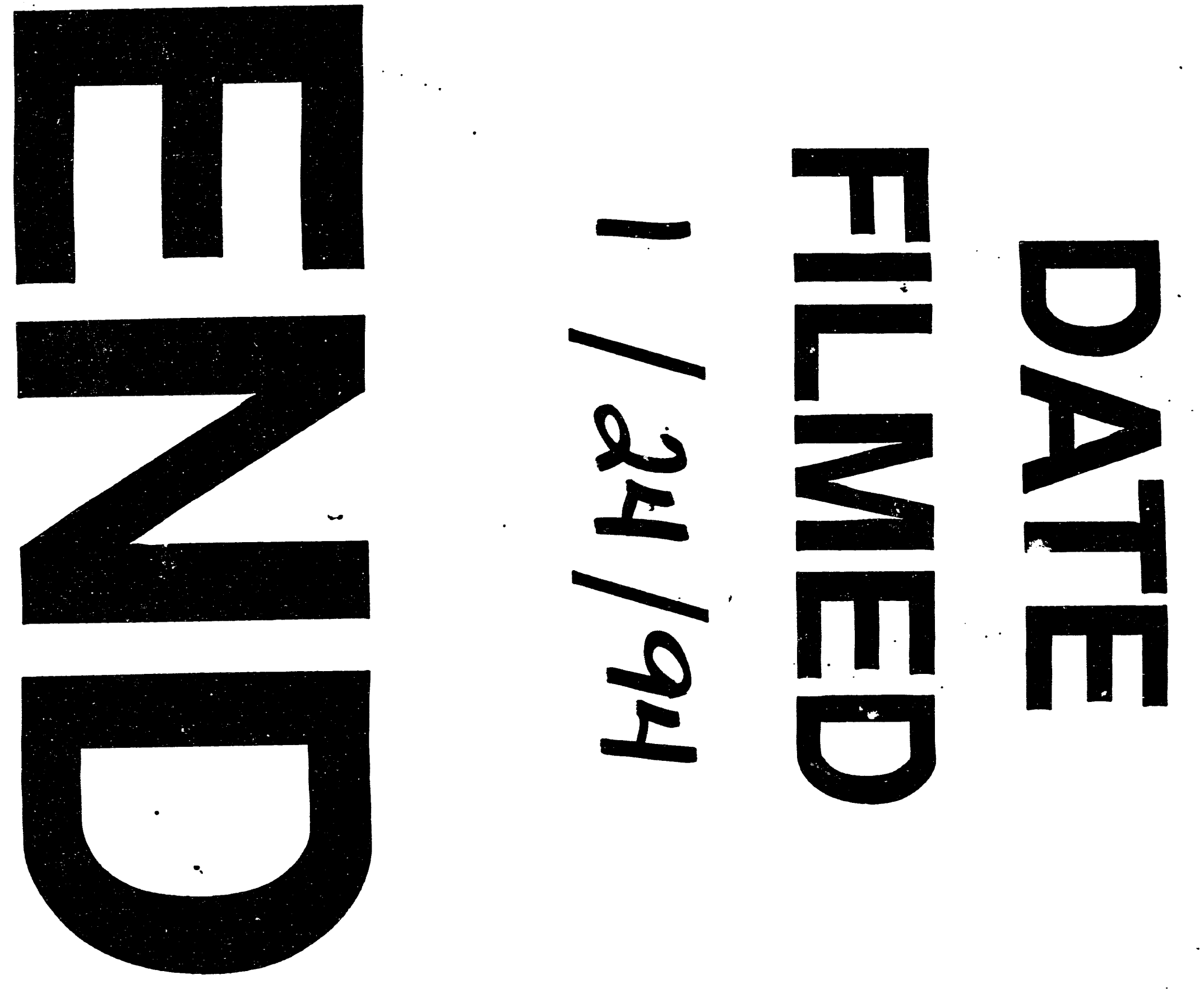